\title{
Supermodularity and Complementarity in Economics: An Elementary Survey
}

\author{
Rabah Amir*
}

\begin{abstract}
The literature on supermodular optimization and games is surveyed from the perspective of potential users in economics. This methodology provides a new approach for comparative statics based only on critical assumptions, and allows a general analysis of games with strategic complementarities. The results are presented in a simplified yet rigourous manner, without reference to lattice theory, for the special case of one-dimensional parameter and actions sets, with the emphasis being on wide accessibility. Detailed applications are presented for well-known models of consumer behavior, monopoly pass-through, Bertrand and Cournot competition, strategic R\&D, search, and matching. Wherever appropriate, useful tricks for applications and comparative comments are inserted.
\end{abstract}

JEL Classification: A23, C60, C72, D43

\section{Introduction}

This survey provides an overview of the theory of supermodular optimization and games, with a marked emphasis on accessibility, for as broad an audience as possible. Supermodular optimization is a new methodology for conducting comparative statics or sensitivity analysis, that is, it determines how changes in exogenous parameters affect endogenous variables in optimizing models. ${ }^{1}$ As such, the use of this methodology is pervasive in economics, and the conclusions thereby derived are often one of the main motivations behind the construction of a model. The main characteristic of this methodology is that it relies essentially on critical assumptions for the desired monotonicity conclusions and dispenses with superfluous assumptions that are often imposed only by the use of the classical method, which is based on the Implicit Function Theorem and includes smoothness, interiority, and concavity. The main insight is indeed quite simple. If, in a maximization problem, the objective reflects a complementarity between an endogenous variable and an exogenous parameter, in the sense that having more of one increases the marginal return to having more of the other, then the optimal value of the former will be increasing in the latter. In the case of multiple endogenous variables, then all of them must also be complements in order to guarantee that their increases are mutually reinforcing. This conclusion follows directly from the underlying complementarity relationship and is thus independent of the aforementioned superfluous assumptions. It thus holds even if there are multiple optimal values of the endogenous variable(s).

Is a new look at complementarity needed? Topkis (1998, p. 3) quotes Samuelson (1947) as asserting the following: "In my opinion the problem of complementarity has received more attention

* Department of Economics, University of Arizona, Tucson, AZ 85721; E-mail: amir@core.ucl.ac.be.

This article has benefitted from comments by, or discussions with Susan Athey, Francis Bloch, Stefano Demichelis, Egbert and Hildegard Dierker, Birgit Grodal, Jon Hamilton, Jean-Francois Merten, Abraham Neyman, David Reiley, John Roberts, Chris Shannon, Xavier Vives, Xavier Wauthy, John Wooders and audiences at CORE, GREQAM-Marseille, the Universities of Arizona, Barcelona (Autonoma), Copenhagen, Rome-LaSapienza, and Stony-Brook.

Received January 2004; accepted January 2004.

${ }^{1}$ This framework of analysis has been developed by Topkis $(1968,1978)$. 
than is merited by its intrinsic importance," only for Samuelson to correct himself later in Samuelson (1974) by adding "The time is ripe for a fresh modern look at the concept of complementarity. The last word has not yet been said on this ancient preoccupation of literary and mathematical economists. The simplest things are often the most complicated to understand fully." It is hoped that this survey will convince the reader of the correctness of Samuelson's latter view.

Another major methodological breakthrough due to this framework of analysis is the theory of supermodular games, better known in economics as games with strategic complementarities. The main characteristic of these games is that they have monotonic reaction curves, reflecting a complementarity relationship between own actions and rivals' actions. As a consequence of Tarski's fixed-point theorem, the latter property guarantees the existence of pure-strategy Nash equilibrium points. Because the latter type of equilibrium is most often the desired concept in economic models, the scope of game-theoretic modeling in economics is thereby substantially enlarged. Another key observation in this respect is that supermodularity is often the relevant notion in the comparative statics of Nash equilibrium points. Furthermore, it will be argued that supermodular games are more conducive to predictable comparative statics properties than games with continuous best-responses, the latter being the other class of games with pure-strategy Nash equilibrium points.

While maintaining rigor in the presentation of the concepts and proofs, some informal aspects in the exposition are adopted, whenever they result in substantial simplification. The major step in achieving such an accessible exposition lies in the restriction of the theory to the case of real action and parameter spaces. While this reduces the scope of the theory and masks its striking elegance, it does, nevertheless, cover most economic applications of broad interest. The main results from the theory of supermodular games are also, thereby, simplified and more accessible. The multidimensional Euclidean framework is presented in summary form at the end of the survey. General comparative comments are given at various points to provide some sense of the scope, usefulness, and limitations of this theory from an applications-oriented perspective. A number of well-known economic applications are covered, including monopoly theory, Cournot and Bertrand competition, a two-stage R\&D model, search, matching, and growth theory. Some of these are covered both with the cardinal and the ordinal notions of complementarity in order to provide some comparative perspective. Various practical tricks in fully exploiting the benefits of this theory are also illustrated via some of the applications presented.

This survey is organized as follows. The next section presents the simplified version of Topkis's Monotonicity Theorem with real decision and parameter spaces and compares this result with the standard method. Section 3 introduces games with strategic complementarities and their key properties, including the comparative statics of their equilibria. Section 4 presents the ordinal complementarity conditions and Milgrom-Shannon's Theorem. The (Euclidean) multidimensional case forms Section 5. Concluding remarks and other aspects of the theory, not covered in this survey, are summarized in Section 6. Last but not least, several illustrative applications and comparative comments are presented throughout to bring out the added value of this new approach in an accessible manner.

\section{Monotone Comparative Statics}

This section provides a simplified exposition of Topkis's (1978) framework in the special case where both the parameter and the decision sets are subsets of the reals. A number of economic applications are then presented to illustrate, in very familiar settings, the relevance and the scope of application of this simplified version of the general theory. 


\section{Topkis's Monotonicity Theorem: The Scalar Case}

Topkis considered the following parameterized family of constrained optimization problems, where $A_{s} \subset A$, with the intent of deriving sufficient conditions on the objective and constraint set that yield monotone optimal solutions:

$$
a^{*}(s)=\arg \max \left\{F(s, a): a \in A_{s}\right\} .
$$

We take the parameter and action sets, $S$ and $A$, to be subsets of $R$, and $A_{s}$ a correspondence from $S$ to $A$, with $A_{s}$ being the set of feasible actions when the parameter is $s$.

A function $F: S \times A \rightarrow R$ has (strictly) increasing differences in $(s, a)$ if

$$
F\left(s^{\prime}, a^{\prime}\right)-F\left(s^{\prime}, a\right)(>) \geq F\left(s, a^{\prime}\right)-F(s, a), \quad \forall a^{\prime}>a, s^{\prime}>s,
$$

or, in other words, if the difference $F\left(\cdot, a^{\prime}\right)-F(\cdot, a)$ is an increasing function. ${ }^{2}$ This property does not discriminate between the two variables in that Equation 2 is clearly equivalent to

$$
F\left(s^{\prime}, a^{\prime}\right)-F\left(s, a^{\prime}\right)(>) \geq F\left(s^{\prime}, a\right)-F(s, a), \quad \forall a^{\prime}>a, s^{\prime}>s .
$$

For functions on $R^{2}$, increasing differences is equivalent to supermodularity, so the two terms will be used interchangeably. ${ }^{3}$

For smooth functions, supermodularity/increasing differences admit a convenient test. ${ }^{4}$

LEMMA 1. If $\mathrm{F}$ is twice continuously differentiable, increasing differences is equivalent to $\partial^{2} F(s$, $a) / \partial a \partial s \geq 0$, for all $a$ and $s$.

PROOF. Increasing differences is equivalent to $F\left(\cdot, a^{\prime}\right)-F(\cdot, a)$ being an increasing function (when $a^{\prime}>a$ ), which is equivalent to $\partial\left[F\left(s, a^{\prime}\right)-F(s, a)\right] / \partial s \geq 0$, or $\left.\partial F\left(s, a^{\prime}\right) / \partial s \geq \partial F(s, a)\right] / \partial s$, that is, $\partial F(s, a) / \partial s$ is increasing in $a$ or $\partial^{2} F(s, a) / \partial a \partial s \geq 0$. $Q E D$.

Increasing differences is interpreted as formalizing the notion of (Edgeworth) complementarity: Having more of one variable increases the marginal returns to having more of the other variable. It turns out that some form of complementarity between endogenous and exogenous variables lies at the heart of any monotone comparative statics conclusion.

A simplified version of Topkis's Monotonicity Theorem is now given. Though a special case of the original result, it is adequate for most applications. It is assumed throughout that $F$ is continuous (or even just upper semi continuous) in $a$ for each $s$, so that the max in Equation 1 is always attained. Furthermore, the correspondence $a^{*}(s)$ then always admits maximal and minimal (single-valued) selections, denoted $\bar{a}(s)$ and $\underline{a}(s)$, respectively.

Theorem 1. Consider Problem 1 with $S, A \subset R$ and assume that

(i) $F$ has increasing differences in $(s, a)$, and

(ii) $A_{s}=[g(s), h(s)]$, where $h, g: S \rightarrow R$ are increasing functions with $g \leq h$.

Then the maximal and minimal selections of $a^{*}(s), \bar{a}(s)$, and $\underline{a}(s)$, are increasing functions. Furthermore, if $(i)$ is strict, then every selection of $a^{*}(s)$ is increasing.

PROOF. By way of contradiction, assume that $\bar{a}(s)$ is not increasing, so that, for some $s^{\prime}>s$, $\bar{a}\left(s^{\prime}\right)<\bar{a}(s)$. Then, using Assumption (ii) and the facts that $\bar{a}(s) \in A_{s}$ and $\bar{a}\left(s^{\prime}\right) \in A_{s^{\prime}}$, we have

\footnotetext{
${ }^{2}$ Throughout, a function $f: S \rightarrow R$ is increasing (strictly increasing) if $x \geq y \Rightarrow f(x) \geq(>) f(y)$.

${ }^{3}$ Supermodularity is defined later on when dealing with multidimensional comparative statics.

${ }^{4}$ Furthermore, if $\partial^{2} f(a) / \partial a_{i} \partial a_{j}>0$, for all $i \neq j$, then $F$ is strictly supermodular. On the other hand, the reverse implication does not quite hold.
} 
$g(s) \leq g\left(s^{\prime}\right) \leq \bar{a}\left(s^{\prime}\right)<\bar{a}(s) \leq h(s) \leq h\left(s^{\prime}\right)$, so that $\bar{a}(s) \in A_{s^{\prime}}$ and $\bar{a}\left(s^{\prime}\right) \in A_{s}$. Now, using the latter facts along with $\bar{a}(s) \in a^{*}(s)$, (i), and $\bar{a}\left(s^{\prime}\right) \in a^{*}\left(s^{\prime}\right)$, we have

$$
0 \geq F\left[s^{\prime}, \bar{a}(s)\right]-F\left[s^{\prime}, \bar{a}\left(s^{\prime}\right)\right] \geq F[s, \bar{a}(s)]-F\left[s, \bar{a}\left(s^{\prime}\right)\right] \geq 0,
$$

so equality holds throughout. Hence, $\bar{a}(s) \in a^{*}\left(s^{\prime}\right)$, a contradiction to the fact that $\bar{a}\left(s^{\prime}\right)=\max \left\{a^{*}\left(s^{\prime}\right)\right\}$, in view of the fact that $\bar{a}\left(s^{\prime}\right)<\bar{a}(s)$. Hence, $\bar{a}(\cdot)$ is increasing. A similar proof holds for $a(\cdot)$.

If Assumption (i) is strict, the same contradiction argument for any selection $\tilde{a}(s)$ of $a^{*}(\cdot)$ shows that Equation 4 holds with a strict middle inequality, a contradiction (as $0>0)$. So $\tilde{a}(\cdot)$ is increasing. $Q E D$.

In this proof, the contradiction hypothesis, that is, $\bar{a}\left(s^{\prime}\right)<\bar{a}(s)$, rules out the possibility that $A_{s} \cap A_{s^{\prime}}=$ $\varnothing$, or, equivalently, that $\sup A_{s}<\inf A_{s^{\prime}}$. Indeed, if the latter were true, then one would necessarily have $\bar{a}\left(s^{\prime}\right)>\bar{a}(s)$, from feasibility alone.

To rephrase the result, in the one-dimensional case with smoothness, it is sufficient for monotone comparative statics that the objective satisfy $\partial^{2} F(s, a) / \partial a \partial s \geq 0$ and the constraint set be a compact interval the end points of which increase in the parameter.

There is an order-dual to Topkis's Theorem, ${ }^{5}$ giving obvious dual conditions under which an argmax is decreasing in a parameter.

Theorem 2. Consider Problem 1 with $\mathrm{S}, \mathrm{A} \subset \mathrm{R}$ and assume that

(i) $F$ has decreasing differences in $(s, a)$, and

(ii) $A_{s}=[g(s), h(s)]$, where $h, g: S \rightarrow R$ are decreasing functions with $g \leq h$.

Then the maximal and minimal selections of $a^{*}(s)$ are decreasing functions. Furthermore, if $(i)$ is strict, then every selection of $a^{*}(s)$ is decreasing.

An alternative way to think of Theorem 2 is that it can be derived from Theorem 1 simply by considering the parameter to be $-s$ instead of $s$. Thus, $F(s, a)$ has increasing differences in $(s, a)$ if and only if $F(s, a)$ has decreasing differences in $(-s, a)$. This connection will prove useful in applications below.

\section{Comparing with Standard Comparative Statics}

For comparative purposes, we now review the standard method for comparative statics taught in graduate economic classes. Consider Problem (1) and assume that $F$ is smooth and the argmax is interior. Then the first-order condition is $F_{2}\left(s, a^{*}(s)\right)=0 .{ }^{6}$ As this is an identity with respect to $s$, under the conditions of the Implicit Function Theorem (here, strong concavity of $F$ in $a$ for each $s$, i.e., $F_{22}<0$ ), we can differentiate them with respect to $s$. Solving for $a^{*}$, one gets (because $F_{22}<0$ by the strong concavity of $F$ in $a$ )

$$
a^{* \prime}(s)=-\frac{F_{21}\left(s, a^{*}(s)\right)}{F_{22}\left(s, a^{*}(s)\right)} \geq 0,
$$

if we assume $F_{21} \geq 0$ (i.e., increasing differences of $F$ in $(s, a)$ ).

Thus Topkis's theorem dispenses with the need for assumptions of concavity and smoothness of the objective function, interiority of the solution and convexity of the feasible set. In not imposing interiority of the solution, Topkis's Theorem requires the constraint set to have the ascendancy property implicit in Condition (ii) of Theorem 1.

\footnotetext{
${ }^{5}$ Writing in the tradition of the operations research literature, Topkis (1978) actually considered the problem of minimizing an objective that has decreasing differences in the action and the parameter. Clearly, to go from his framework to the present one or vice versa, one simply needs to multiply the objective by -1 .

${ }^{6}$ Throughout, subscripts denote partials with respect to the corresponding variable.
} 
This condition guarantees that the boundaries of the constraint set are increasing functions of the parameter, so that an argmax that lies on parts of the boundary of the feasible set for some parameter values would clearly inherit the desired monotonicity property, even if it switches back and forth between the interior of the feasible set and its boundary. In a nutshell, Topkis's Theorem is able to dispense with concavity requirements because, if these fail locally, the argmax will be on the boundary, and hence, increasing in the parameter by Condition (ii).

The traditional comparative statics method only works when there is a unique argmax that varies smoothly with the parameter without ever straying against the constraint. As a result, traditional economic theory inherited an excessive tendency to impose assumptions of concavity of objective functions, Inada-type conditions, and convexity of feasible sets even in cases where these are not readily justifiable on economic grounds. Likewise, the development of theories where important nonconvexities are inherently present, due, for instance, to some form of increasing returns, was probably hampered or delayed. In other words, the scope and direction of economic research were probably negatively influenced by these important limitations of a purely methodological nature.

Another key advantage of this new method, not covered here, is that it allows the parameter set to be any partially ordered set, such as a set of integers, ${ }^{7}$ or such abstract objects as a set of probability distributions partially ordered, for example, by first-order stochastic dominance ${ }^{8}$ or a set of sets ordered by inclusion. It also allows the decision set similar latitude.

\section{Economic Applications}

We now provide several simple illustrations of Topkis's Theorem drawn from various familiar settings in economics. While all of these examples demonstrate natural ways in which complementarity can arise and be exploited for comparative statics, some of the examples also serve to illustrate useful tricks in using this methodology. In particular, a monotone-transformed objective function may satisfy the conditions of Topkis's Theorem under some natural assumptions while obviously preserving the same optimal argmax. Also, the same machinery can be used to perform nonmonotone comparative statics on a change of the decision variable. The most frequent change of variable is to consider minus the original variable, allowing one to go back and forth between increasing and decreasing outcomes. To check the conditions of the Theorem, the cross-partial test will always be used due to its convenience, although smoothness is actually not needed for most of the results presented.

\section{Consumer Theory}

Consider the familiar problem of a consumer maximizing utility $U\left(x_{1}, x_{2}\right)$ from the consumption of two goods $x_{1}$ and $x_{2}$ at given respective prices $p_{1}$ and $p_{2}$ and income $m$. We wish to derive conditions under which $x_{1}$ is a normal good or the demand for $x_{1}$ increases in $m$. The problem is then $\max \left\{U\left(x_{1}, x_{2}\right): p_{1} x_{1}+p_{2} x_{2}=m\right\}$. If $U$ is increasing in $x_{2}$, we can solve the constraint for $x_{2}$ and plug it into the objective,

\footnotetext{
${ }^{7}$ In deriving the comparative statics properties of Cournot equilibria, Amir and Lambson (2000) show that the simplification of treating the number of firms as a real number—common in the literature, as in Seade (1980)—can lead to misleading or erroneous results.

${ }^{8}$ Thus, a comparative statics conclusion may well take the form that as social inequality increases (exogenously) according to some nonparametric measure of income distribution, then crime (endogenously) increases, or as an agent's market information improves in some nonparametric way, she invests more, and so on.
} 


$$
\max \left\{U\left(x_{1},\left(m-p_{1} x_{1}\right) / p_{2}\right): x_{1} \in\left[0, m / p_{1}\right]\right\} .
$$

The constraint set $\left[0, m / p_{1}\right]$ clearly satisfies Assumption (ii) of Theorem 1 , with $g$ being the 0 function and $h$ the function $m / p_{1}$. By Lemma 1 , assuming $U$ is smooth, the objective in Equation 5 has increasing differences in $\left(x_{1}, m\right)$ if and only if the familiar condition

$$
p_{2} U_{21}\left(x_{1}, x_{2}\right)-p_{1} U_{22}\left(x_{1}, x_{2}\right) \geq 0, \quad \forall x_{1}, x_{2} \geq 0
$$

holds. It follows from Topkis's Theorem that Condition 6 is sufficient to guarantee that $x_{1}$ is a normal good. Observe that this conclusion survives if income is expressed in discrete units, or if demand $x_{1}^{*}(m)$ switches from being interior to being on a boundary as $m$ changes, or if demand is multivalued for some income levels (the conclusion that $x_{1}$ is a normal good then holds for the extremal selections of $x_{1}^{*}(m)$ ). Furthermore, no concavity-type assumptions on $U$ are needed! In fact, the only assumptions needed here are Condition 6 and the fact that the utility function is increasing in the consumption of $x_{2}$ (in solving the budget constraint).

\section{Monopoly Pass-Through}

Consider a monopoly firm with constant unit cost $c$ charging a price $p \in[c, \infty)$ and facing a direct demand function $D(\cdot)$. The profit function is

$$
\Pi(p, c)=(p-c) D(p), \quad p \in[c, \infty) .
$$

To verify increasing differences of the profit function directly, we have $\partial^{2} \Pi(p, c) / \partial p \partial c=-D^{\prime}(p)$, which is $\geq 0$ iff $D^{\prime}(p) \leq 0$. Because the feasible set $[c, \infty)$ has the form specified in Theorem 1 , assuming $D^{\prime}(p) \leq 0$ is sufficient for the conclusion that the extremal selections from the optimal price $p^{*}$ are increasing in $c$.

We now present an alternative way to arrive at the same conclusion. As the optimal price is invariant under a monotone transformation, we may equivalently consider the objective

$$
\log \Pi(p, c)=\log (p-c)+\log D(p), \quad p \in[c, \infty) .
$$

As $\partial^{2} \log \Pi(p, c) / \partial p \partial c=(p-c)^{-2} \geq 0$, the desired monotonicity conclusion follows. Observe here that $D$ need not be decreasing in own price for this conclusion to hold! ${ }^{9}$

We now present interesting comparative statics results of a nonmontone sort, simply by making use of a change of variable. Define mark-up (over marginal cost) as $m \triangleq p-c$ and write the equivalent objective with this change of variable as

$$
\log \tilde{\Pi}(m, c) \triangleq \log (m)+\log D(m+c) .
$$

The latter objective has decreasing differences in $(m, c)$ if $D$ is $\log$-concave (i.e., $\log D$ is concave). ${ }^{10}$ Because the constraint set is $[0, \infty)$, it follows from Topkis's Theorem that the maximal and minimal selections from the optimal mark-up $m^{*}(c)$ are decreasing in $c$ or, equivalently, that the extremal selections of $p^{*}$ have all their slopes $\leq 1$ (because $\left.p^{*}(c)=m^{*}(c)+c\right)$. Overall then, $p^{*}$ has all its slopes in $[0,1]$ and is thus continuous and single-valued. Hence, there is always positive but partial pass-through.

\footnotetext{
9 Observe here that taking monotone transformation of a nonsupermodular objective function may bring about supermodularity of the transformed objective. We will say more on this point later.

${ }^{10}$ Indeed, $\partial^{2} \log D(m+c) / \partial m \partial c=\left[D D^{\prime \prime}-D^{\prime 2}\right] / D^{2} \geq 0$ if and only if $D D^{\prime \prime}-D^{\prime 2} \geq 0$, that is $D$ is $\log$ concave.
} 
If $D$ is log-convex, a similar argument shows that $\log \tilde{\Pi}(m, c)$ has increasing differences in $(m, c)$, so that the extremal selections of $m^{*}(c)$ are increasing in $c$ or, equivalently, the extremal selections of $p^{*}$ have all their slopes $\geq 1$, so that pass-through exceeds $100 \%{ }^{11}$

\section{Assortative Matching}

Becker (1973) considered a model of marriage with $n$ women and $n$ men looking to match to form $n$ couples. Each of the sexes is ranked by productive ability according to the natural order in $\{1$, $2, \ldots, n\}$, with person 1 being the least productive and person $n$ the most productive. If woman $i$ and man $j$ get married, they generate a surplus of $f(i, j)$ as a couple. A matching is any list of $n$ couples consisting of one woman and one man each. The social planner's problem is to find a matching that maximizes social surplus, defined as the sum $\sum f(i, j)$ over all couples in a given matching. Becker's main question is to derive conditions on $f$ that lead to assortative matching, that is, to the couples being $(1,1),(2,2), \ldots,(n, n)$. He shows that, if $f$ has strictly increasing differences, ${ }^{12}$ the optimal matching must be assortative. For otherwise, there would necessarily be two couples $(i, j)$ and $\left(i^{\prime}, j^{\prime}\right)$ with (say) $i^{\prime}>i$ but $j^{\prime}<j$ as part of the presumed matching, so that by increasing differences of $f$, there would hold $f\left(i^{\prime}, j\right)+f\left(i, j^{\prime}\right)>f(i, j)+f\left(i^{\prime}, j^{\prime}\right)$, which contradicts the optimality of the presumed matching, as it says that matching $i^{\prime}$ to $j$ and $i$ to $j^{\prime}$ leads to a higher surplus than $i$ to $j$ and $i^{\prime}$ to $j^{\prime}$.

\section{Growth Theory}

Consider a two-period version of the standard Solow-Koopmans optimal growth model with possible increasing returns. Assume the utility function $u$ is such that $u^{\prime}>0$ and $u^{\prime \prime}<0$, the production function $f$ is such that $f^{\prime}>0$ (no restrictions on $f^{\prime \prime}$ ), and the discount factor $\delta \in(0,1)$. With $y_{t}$ denoting savings, the planner's objective is

$$
\max \sum_{t=1}^{2} u\left(x_{t}-y_{t}\right) \quad \text { subject to } x_{t+1}=f\left(y_{t}\right) \quad \text { and } \quad y_{t} \in\left[0, x_{t}\right] \text {. }
$$

The two-period value function $V_{2}$ satisfies

$$
V_{2}(x)=\max \{u(x-y)+\delta u[f(y)]: y \in[0, x]\} .
$$

Here, the maximand need not be concave in $y$, so $y^{*}(x)$ may be multivalued. Because the maximand has increasing differences in $(x, y)$ and the constraint set satisfies Condition (ii) of Theorem 1 , it follows that the optimal savings correspondence $y^{*}(x)$ is increasing in $x$ (no restrictions are actually needed on $f$ ).

For an infinite-horizon extension of this example, where the parameter set includes $\delta$ and the length of the horizon, see Amir, Mirman, and Perkins (1991) and Amir (1996a).

Many more examples are given in the next section in one of the most important general uses of Topkis's Theorem: When showing the best response map in a supermodular game is increasing in the rivals' strategies (viewing the latter as a parameter).

\footnotetext{
${ }^{11}$ As examples, consider two of the most widely used demand functions. For linear demand (clearly log concave), cost pass through is $50 \%$, and for constant-elasticity demand (log convex), pass through is $\varepsilon(1+\varepsilon)^{-1}>1$. The above results are a generalization of this illustration.

12 The interpretation of increasing differences in this context is the standard one: The productive abilities of the two members of the couple are complements in production, or that having a higher ability mate increases a person's marginal productivity of own ability.
} 


\section{Supermodular Games}

A fundamental contribution of the methodology at hand is that it gave rise to a new class of games, introduced by Topkis (1979) and further developed by Vives (1990) and Milgrom and Roberts (1990b), which always have pure-strategy equilibrium points in addition to many other nice general properties. The importance of this class of games in economics is underscored by the fact that many standard models of oligopoly and macroeconomic coordination games are supermodular games under quite general - though not universal — conditions on their primitives. The class of supermodular games, also known as games with strategic complementarities, substantially enlarges the scope of application of noncooperative game theory in economics, particularly because it dispenses with the reliance on mixedstrategies to ensure existence of Nash equilibrium, randomized behavior still being a controversial feature of game theory for economists. Recall that the classical approach to existence of pure-strategy Nash equilibrium generally requires the quasi-concavity of each payoff function in own action. ${ }^{13}$

\section{The Class of Supermodular Games}

An $n$-player normal-form game is given by a tuple $\left(N, A_{i}, F_{i}\right)$, where $A_{i} \subset R, N=\{1,2, \ldots, n\}$ is the player set and $A_{i}$ and $F_{i}: A_{1} \times A_{2} \times \cdots A_{n} \rightarrow R$ are player $i$ 's action set and payoff function. Such a game is supermodular if, for every player, (i) the action set is a compact set and (ii) each payoff function has increasing differences in own action and in each rival's action. ${ }^{14}$

The key property of supermodular games is an immediate consequence of Topkis's Theorem: For each player, the best-reply (or reaction) correspondence, mapping the rivals' action vector $a^{-i}$ vector into the player's set of best responses has extremal selections that are increasing in each rival's action. Thus, the overall best response mapping, taking the full-action vector $a$ into the set of best responses, also has extremal selections that are increasing (coordinatewise). That either one of these selections has a fixed point-which is clearly a pure-strategy equilibrium of the game-is a direct consequence of Tarski's (1955) fixed point theorem stated without proof next.

Theorem 3. Let $A$ be a compact Euclidean interval and $F: A \rightarrow A$ be increasing. Then the set of fixed points of $F$ is nonempty and has (coordinatewise) largest and smallest elements.

In the one-dimensional case, Tarski's Theorem is easily illustrated graphically, say for a map from $[0,1]$ to $[0,1]$. Then, considering the mapping $G(a) \triangleq F(a)-a$, one has $G(0)=F(0) \geq 0$ and $G(1)=F(1)-1 \leq 0$. Clearly, the graph of $G$ can only have upward jumps, so for it to get from above to below the horizontal axis, it must necessarily cross it at some point $\bar{a}$, for which, then, $G(\bar{a})=\bar{a}$.

An $n$-player submodular game is defined in a dual way, the only change being that decreasing differences replaces increasing differences. By Theorem 2, the reaction correspondences of a submodular game are downward sloping. ${ }^{15}$ Because there is no version of Tarski's Theorem for downward-sloping mappings (a fact that is easy to see graphically), submodular games need not have pure-strategy equilibrium points. An interesting exception is the two-player case $(n=2)$. If, in a submodular game, we think of (say) player 2's action vector as being $-a_{2}$ instead of $a_{2}$, then the

\footnotetext{
${ }^{13}$ Additionally, because existence is then established via the Brouwer-Kakutani fixed-point Theorem, the strategy spaces have to be convex, and the best-response correspondence upper hemicontinuous (with the latter requiring some form of continuity of the payoff in the actions).

${ }^{14}$ Each payoff must also be upper semicontinuous in own action to ensure that the best response of each player is well defined (i.e., that the maximum is attained).

15 Thus, supermodular games and submodular games correspond to games with strategic complementarity and substitutability, respectively, in the now-standard language of Bülow, Geanakoplos, and Klemperer (1985).
} 
game becomes a supermodular game because decreasing differences in $\left(a_{1}, a_{2}\right)$ become increasing differences in $\left(a_{1},-a_{2}\right)$, as can be easily seen. ${ }^{16}$ No such argument holds for $n \geq 3$.

\section{Coincidence of Solution Concepts}

For any normal-form game, let $U \subset A_{1} \times A_{2} \times \cdots A_{n}$ denote the set of strategies that survive iterated deletion of strictly dominated strategies. It is well known that only pure strategies in $U$ can have positive mass at any mixed-strategy or correlated-strategy equilibrium (see, e.g., Fundenberg and Tirole 1991).

Because the best-response map of a supermodular game is increasing, it is easy to see that there exist (coordinatewise) largest and smallest Nash equilibria. Denote these by $\bar{a}$ and $\underline{a}$. The following is a simplified version of a key result in Milgrom and Roberts (1990b):

THEOREM 4. For any supermodular game, sup $U=\bar{a}$ and inf $U=\underline{a}$.

Proof. Let $\mathbf{0}$ and $\mathbf{1}$ stand for the smallest and the largest action vectors of all player 1's (say) rivals. We first show for player 1 that the largest best reply to $\mathbf{1}$, denoted $r_{1}(\mathbf{1})$, strictly dominates all larger actions. To this end, by increasing differences of $F_{1}$, for any action vector $a_{-1}$ of the rivals and any $a_{1}>r_{1}(\mathbf{1})$, we have

$$
F_{1}\left(a_{1}, a_{-1}\right)-F_{1}\left(r_{1}(\mathbf{1}), a_{-1}\right) \leq F_{1}\left(a_{1}, \mathbf{1}\right)-F_{1}\left(r_{1}(\mathbf{1}), \mathbf{1}\right)<0,
$$

because $r_{1}(\mathbf{1})$ is the largest best reply to $\mathbf{1}$. Hence, $r_{1}(\mathbf{1})$ strictly dominates all actions $a_{1}>r_{1}(\mathbf{1})$.

A similar argument shows that $r_{1}(\mathbf{0})$, defined as the smallest best reply to $\mathbf{0}$, strictly dominates all actions $a_{1}<r_{1}(\mathbf{0})$.

Now, the first step of the iteration is to eliminate all player 1's actions that are $>r_{1}(\mathbf{1})$ or $r_{1}(\mathbf{0})$. Repeat this procedure for every other player, and then again starting with player 1 , and so on. As shown in Figure 1, this process will clearly converge to $[\underline{a}, \bar{a}]$. $Q E D$.

Thus, $\underline{a}$ and $\bar{a}$ form the same bounds on most solution concepts for normal-form games, including pure, mixed, or correlated-strategy equilibrium. When there is a unique pure-strategy equilibrium, more can be said.

Corollary 1. A supermodular game with a unique pure-strategy equilibrium, $a^{*}$, is dominancesolvable, and $a^{*}$ is also the unique mixed-strategy equilibrium and correlated equilibrium.

PRoOF. This result follows from Theorem 4 because $\underline{a}=\bar{a}=a^{*}=U$ here, along with the fact that action profiles outside of $U$ cannot be part of a mixed or correlated equilibrium. $Q E D$.

The intuition behind this result can be captured by a simple graphical illustration in the case of twoplayer games. In Figure 1a, one round of deletion of strategies that are not best replies to any strategy of the rival is shown. Continuing this process, starting at each round from the reduced interval of actions left from the previous round, would clearly converge to the unique equilibrium. In Figure $1 \mathrm{~b}$, in view of the multiplicity of pure-strategy equilibria, the same process will clearly converge to the rectangle $[\underline{a}, \bar{a}]$.

\section{Comparative Statics of Equilibrium Points}

To determine the direction of change of an equilibrium point as an exogenous parameter changes, Topkis's Theorem is not a priori applicable. Consider a parametric family of games with

\footnotetext{
${ }^{16}$ An alternative argument, due to Vives (1990), is as follows. For a submodular game, the extremal reaction curves $r_{1}$ and $r_{2}$ are both decreasing, so the composition $r_{1} \circ r_{2}: A_{1} \rightarrow A_{1}$ is increasing. By Tarski's theorem, the latter has a fixed point, $a_{1}^{*}$. Then $\left(a_{1}^{*}, r_{2}\left(a_{1}^{*}\right)\right)$ is easily seen to be a Nash equilibrium.
} 

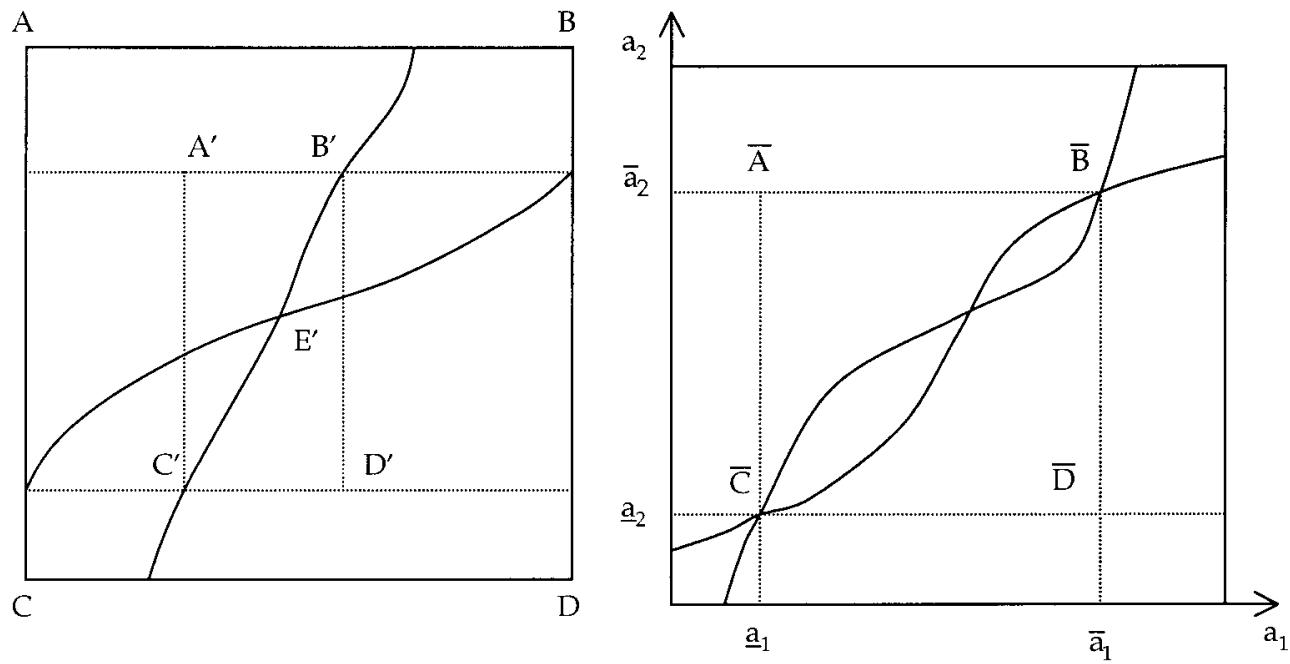

Figure 1. (a) The first step of the process shrinks the joint strategy set from $A B C D$ to $A^{\prime} B^{\prime} C^{\prime} D^{\prime}$. This process converges to $E$. (b) The same process will converge to $\bar{A} \bar{B} \bar{C} \bar{D}$ here.

Player $i$ 's payoff given by $F_{i}\left(a^{i}, a^{-i}, s\right)$ where $s \in S$ is a parameter. Milgrom and Roberts (1990b) prove the following very useful result in applying the theory of supermodular games:

Theorem 5. Assume that (i) for each $s \in S \subset R$, the game is supermodular, and (ii) $F_{i}$ has increasing differences in $\left(a^{i}, s\right)$ for each $a^{-i}$. Then the extremal equilibria of the game are increasing functions of $s$.

PRoof. Let $B_{s}(\cdot): A \rightarrow A$ denote the best-reply correspondence when the parameter is $s$. For each $s \in S$, there are smallest and largest Nash equilibria, by the supermodularity assumption. Let $s_{1}>s_{2}$ and $\bar{a}(s)$ denote the largest Nash equilibrium of the game with parameter $s, s \in S$. For each $i$, player $i$ 's reaction correspondence $r_{i}^{s}\left(a^{-i}\right)$ shifts out as $s$ increases, by the increasing differences assumption and Topkis's Theorem. Hence, $B_{s}(\cdot)$ also shifts out as $s$ increases. Because $\bar{a}\left(s_{2}\right)$ is the largest fixed point of $B\left(s_{2}\right)$ and because $B_{s_{1}}(\cdot) \geq B_{s_{2}}(\cdot), B_{s_{1}}(\cdot)$ maps $\left[\bar{a}\left(s_{2}\right), 1_{A}\right]$ into itself. By Tarski's Theorem, the latter restricted map has a fixed point in $\left[\bar{a}\left(s_{2}\right), 1_{A}\right]$, which is then clearly $\geq \bar{a}\left(s_{2}\right)$.

A similar argument works for the smallest equilibrium $\underline{a}(s)$. $Q E D$.

Thus, for a supermodular game with smooth payoffs, the extremal equilibria increase in the parameter if $\partial^{2} F_{i} / \partial a^{i} \partial s \geq 0$ for all $i$. The intuition behind this result is nicely captured by Figure 2 . Indeed, from Topkis's Theorem applied at fixed $\mathrm{a}^{-i}$, the effect of Theorem 5 is precisely that player $i$ 's reaction correspondence shifts up as $s$ increases. It follows that the maximal and minimal equilibrium points both increase, while the middle equilibrium moves in the opposite direction, in the case of 3 equilibria. ${ }^{17}$ The overall increase in the (extremal) equilibrium actions for each player can intuitively be regarded as consisting of two effects. The direct effect is the positive response of the player's action to the increase in the parameter, or the shift in his reaction correspondence. The indirect effect is the positive reaction of the player to the direct-effect increases of the other players' actions.

${ }^{17}$ Under multiple equilibria, it has long been known that the comparative statics properties of equilbria are intimately linked to their stability properties (say, in terms of Cournot best-reply dynamics). This is Samuelson's (1947, 1974) so-called Correspondence Principle. The connection with Theorem 5 is that the extremal equilbria of a supermodular game are always stable. See Echenique (2002) for a recent thorough study. 


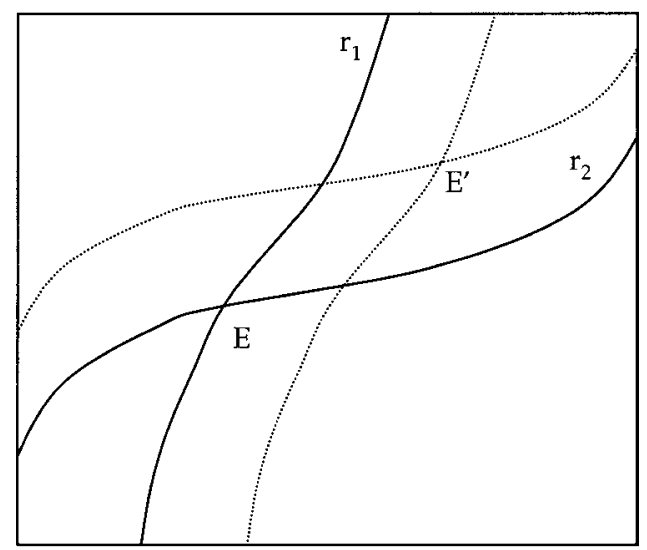

Figure 2. As the Reaction Curves Shift from the Solid Lines to the Dotted Lines, the Nash Equilibrium Shifts from $E$ to $E^{\prime}$

This theorem has natural applications in a variety of settings in economic theory, some of which are explored below. It confirms that strategic complementarity of a game is a natural property that is easily compatible with clear-cut comparative statics properties.

By contrast, a game with a continuous (single-valued) best-response mapping but without strategic complementarities need not possess clear-cut monotone comparative statics properties, as is easily seen graphically. In other words, outward shifts in the continuous reaction curves need not imply higher equilibrium actions unless the game is symmetric. Observe that the requisite fact that the reaction curves shift out as the parameter increases is a consequence of the fact that each payoff has increasing differences in own action and in the parameter. Thus, supermodularity plays a role in comparing equilibrium points even within the classical paradigm. Generally, with continuous reaction curves, the equilibrium will satisfy continuity in the parameter, a property that guarantees a sense of stability in that equilibria will not vary drastically for small parameter variations. However, in most cases in economics, one is actually interested in qualitative comparative statics and wishes to determine a monotone direction of response of endogenous variables to variations in the exogenous variables. In most exercises of comparative equilibrium points in applications, supermodularity is most often (at least tacitly) behind the analysis.

There is no dual version of Theorem 5 for submodular games, or games with strategic substitutes, even in the two-player case. Indeed, if one only replaces "supermodular" by "submodular" in Theorem 5 , the analogous reasoning would tell us that the (then downward-sloping) reaction correspondences also shift outward as the parameter increases, but here it need not follow that the equilibrium actions will both increase, as can easily be seen from the analogous picture. It all depends on the relative magnitudes of the shifts of the two curves. In this case, a similar intuitive break-up of the overall response into a direct and an indirect effect will reveal that the two effects are conflicting, with the direct effect being the same as the above case and the indirect effect amounting to a decrease in the player's action. The overall effect then depends on the relative strengths of these two effects, hence the ambiguous final outcome.

\section{Other Important Properties}

Supermodular games possess other properties of interest, which have not been addressed here. An important property of supermodular games is that general learning schemes, so-called adaptive learning schemes, ${ }^{18}$ converge to the interval bounded by the extremal pure-strategy equilibria (Topkis

\footnotetext{
${ }^{18}$ This is a very broad class of learning schemes that includes the well-known special cases of Cournot best-reply dynamics and
} fictitious play. 
1979; and Milgrom and Roberts 1990b; Vives 1990). This property has also been used by Topkis (1979) to devise algorithms for computing equilibria of supermodular games. Echenique (2001b) shows that a supermodular game with real action sets is also supermodular in mixed strategies when these are ordered with first-order stochastic dominance. Echenique and Edlin (2001) establish that mixed-strategy equilibria of supermodular games are unstable with respect to a large class of dynamics (as in coordination games, such as the Battle of the Sexes). For Bayesian games, such as some classes of auctions, an extension (Vives 1990) and an exploitation (Athey 2001) of the property of strategic complementarity are used to establish existence of Bayesian equilibrium. Finally, Echenique (2001a) shows that the class of supermodular games can be substantially enlarged if the partial order on the strategy sets is endogenous, as opposed to being fixed a priori.

\section{Examples of Supermodular Games}

We now provide several examples of well-known models in economic theory that are supermodular games under natural conditions.

\section{Coordination Games}

The standard $2 \times 2$ Battle of the Sexes given below is a supermodular game because $2+1>0+0$. More generally, large classes of coordination-type games are typically supermodular games.

\begin{tabular}{|l|l|}
\hline 0,0 & 2,1 \\
\hline 1,2 & 0,0 \\
\hline
\end{tabular}

\section{Search}

In Diamond's (1982) model, agent $i$ expands effort $a^{i} \in[0,1]$ searching for trading partners, and has a payoff function given by (with $s>0$ being a parameter characterizing the search environment)

$$
F_{i}\left(a^{i}, a^{-i}\right)=s a^{i} \sum_{j \neq i} a^{j}-C_{i}\left(a^{i}\right) .
$$

Because $\partial^{2} F_{i}\left(a^{i}, a^{-i}\right) / \partial a^{i} \partial a^{j}=s>0$ for all $i \neq j$, the game is supermodular for any cost functions. Furthermore, because $\partial^{2} F_{i} / \partial a^{i} \partial s \geq 0$, it follows from Theorem 4 that the largest and the smallest equilibria are increasing in $s$ (which is a measure of the ease of search).

\section{Bertrand Oligopoly}

In price competition with differentiated products and linear costs, firm $i$ 's profit function, when it charges price $p^{i}$ and its rivals' price vector is $p^{-i}$, is

$$
F_{i}\left(p^{i}, p^{-i}\right)=\left(p^{i}-c_{i}\right) D_{i}\left(p^{i}, p^{-i}\right),
$$

where $c_{i}$ is its unit cost and $D_{i}$ its demand function. ${ }^{19}$ We can clearly restrict consideration to the price set $\left[c_{i}, \infty\right)$ for firm $i$, because prices in $\left[0, c_{i}\right)$ are dominated strategies. Because monotone transformations leave the best-reply structure unchanged and

$$
\log F_{i}\left(p^{i}, p^{-i}\right)=\log \left(p^{i}-c_{i}\right)+\log D_{i}\left(p^{i}, p^{-i}\right),
$$

\footnotetext{
${ }^{19}$ It is assumed that firms satisfy whatever demand is forthcoming, that is, that there is no rationing.
} 
it follows (Milgrom and Roberts 1990b) that the game is $\log$-supermodular if $\log D_{i}$ has increasing differences in $\left(p^{i}, p^{-i}\right)$ or, equivalently, using the cross-partial test, if, for all $j \neq i$,

$$
D_{i}\left(p^{i}, p^{j}\right) \frac{\partial^{2} D_{i}\left(p^{i}, p^{j}\right)}{\partial p^{i} \partial p^{j}}-\frac{\partial D_{i}\left(p^{i}, p^{j}\right)}{\partial p^{j}} \frac{\partial D_{i}\left(p^{i}, p^{j}\right)}{\partial p^{j}} \geq 0 .
$$

The latter property has an intuitive and precise interpretation: Firm $i$ 's price elasticity of demand is increasing in the rivals' prices. Most demand functions used in industrial economics satisfy this condition. Many examples are provided in Vives (1999) and Milgrom and Roberts (1990b), all of which lead to a unique pure-strategy equilibrium, so that by Corollary 1, the Bertrand oligopoly is then dominance solvable.

Next, we illustrate the application of the comparative-equilibrium result. Because $\log \left(p^{i}-c_{i}\right)$ clearly has increasing differences ${ }^{20}$ in $\left(p^{i}, c\right)$, where $c$ is the vector of unit costs, it follows from Theorem 5 that, if $\log D_{i}$ has increasing differences, the largest and smallest equilibrium prices are nondecreasing functions of $c$, that is, that higher costs from any subset of the firms result in all firms' prices being higher and are thus always passed on to consumers. ${ }^{21}$

\section{Cournot Duopoly}

For homogeneous-good quantity competition, if firm $i$ produces output level $q_{i}$ at a $\operatorname{cost} C_{i}\left(q_{i}\right)$ and $P$ is the inverse demand function, firm $i$ has profit

$$
F_{i}\left(q_{1}, q_{2}\right)=q_{i} P\left(q_{1}+q_{2}\right)-C_{i}\left(q_{i}\right)
$$

Because with $P^{\prime} \leq 0$, we have

$$
\partial^{2} F_{i}\left(q_{1}, q_{2}\right) / \partial q_{1} \partial q_{2}=P^{\prime}\left(q_{1}+q_{2}\right)+q_{1} P^{\prime \prime}\left(q_{1}+q_{2}\right) \leq 0 \text { for all } q_{1}, q_{2} \geq 0,
$$

if and only if

$$
P^{\prime}(z)+z P^{\prime \prime}(z) \leq 0 \text { for all } z \geq 0,
$$

it follows that the game is submodular if the latter condition holds (see Novshek 1985; Amir 1996b). This conclusion is easily seen to be valid even in the $n$-firm case, for all $n .^{22}$

Furthermore, for $n=2$, if one thinks of (say) firm 2's decision as being $-q_{2}$ instead of $q_{2}$, then, under the above condition on demand, we clearly have $\partial^{2} F_{i}\left(q_{1}, q_{2}\right) / \partial q_{1} \partial\left(-q_{2}\right) \geq 0, i=1,2$, so that the Cournot duopoly is then a supermodular game. This order-reversing trick works for any two-player submodular game but does not work for three or more firms, that is, in general, a submodular $n$-player game is also a supermodular game if and only if $n=2$. For a generalization to differentiated products, see Hoerning (2003).

\section{$R \& D$ Competition}

Consider the following variant, due to Amir and Wooders $(1999,2000)$, of the standard twostage game of R\&D with one-way spillovers and product market competition. The latter is modeled in

\footnotetext{
${ }^{20}$ Indeed, $\partial^{2} F_{i}(p) / \partial p^{i} \partial c_{i}=\partial^{2} \log \left(p^{i}-c_{i}\right) / \partial p^{i} \partial c_{i}=1 /\left(p^{i}-c_{i}\right)^{2} \geq 0$, and $\partial^{2} F_{i}(p) / \partial p^{i} \partial c_{j}=0$ for $j \neq i$.

${ }^{21}$ A firm whose unit cost increases will increase its price via the direct effect of responding to its own cost increase and via the indirect effect of responding to rivals' cost increases. A firm whose unit cost remains constant will still increase its price but only via the latter effect.

${ }^{22}$ Exploiting in a crucial way the special structure of the Cournot model, that a firm's profit only depends on its output and on the aggregate output of the rivals, Novshek (1985) showed that a submodular Cournot oligopoly always has a pure-strategy Cournot equilibrium, even though the game is then not supermodular.
} 
reduced form by assuming a unique equilibrium in the second stage with equilibrium payoff to firm 1 (say) given by $\Pi\left(c_{1}, c_{2}\right)$, where $c_{1}$ and $c_{2}$ are the post-R\&D constant unit costs for firms 1 and 2 . Denoting their first-period decisions, their R\&D cost reductions, by $x_{1}$ and $x_{2}$, their postspillover cost reductions are, respectively (assuming without loss of generality that $x_{1} \geq x_{2}$ ): $X_{1}=x_{1}$, and $X_{2}=x_{1}$ with probability $\beta$, and $x_{2}$ with probability $(1-\beta)$. Thus, $\beta$ here is the probability that a full spillover takes place, only from the firm ahead in $\mathrm{R} \& \mathrm{D}$ to the laggard. With $c$ and $f$ denoting the common initial unit cost and the R\&D cost function, respectively, firm 1's prespillover expected payoff is

$$
\left\{\begin{array}{ll}
\beta \Pi\left(c-x_{1}, c-x_{1}\right)+(1-\beta) \Pi\left(c-x_{1}, c-x_{2}\right)-f\left(x_{1}\right), & \text { if } x_{1} \geq x_{2} \\
\beta \Pi\left(c-x_{2}, c-x_{2}\right)+(1-\beta) \Pi\left(c-x_{1}, c-x_{2}\right)-f\left(x_{1}\right), & \text { if } x_{1} \leq x_{2}
\end{array} .\right.
$$

Assuming $\Pi$ is strictly submodular, $\Pi_{1}<0, \Pi_{2}>0$, and $\Pi(x, x)$ is decreasing ${ }^{23}$ in $x$, Amir and Wooders (2000) show that the above payoff is submodular ${ }^{24}$ in $\left(x_{1}, x_{2}\right)$, so that the last paragraph of the Cournot Duopoly example applies to the present duopoly as well. They also prove that (i) this game always has asymmetric equilibria, but no symmetric equilibria (though the firms are exante identical), ${ }^{25}$ and (ii) although firm $i$ 's profit is submodular in $\left(x_{i}, \beta\right)$, so that optimal reactions shift inward as $\beta$ increases, the equilibrium R\&D levels need not both decrease in $\beta$ (see Amir and Wooders 1999).

\section{Ordinal Complementarity Conditions}

The properties of supermodularity and increasing differences are clearly of a cardinal nature. They need not be preserved by monotone transformations of the objective function. Yet the monotonicity of the argmax clearly survives such transformations, a property of which the usefulness has already been illustrated in our applications above. For instance, in the monopoly example of section 2, it was shown that, while increasing differences of profits require the assumption that demand be nondecreasing in price, increasing differences of log profits hold without any assumption. This illustrates the possibility that applying a judicious monotone transformation to an objective function may change the comparative statics analysis. The question then naturally arises as to whether an ordinal version of the property of increasing differences could be developed (that would be invariant under monotone transformations).

\section{The Milgrom-Shannon Theorem}

Milgrom and Shannon (1994) provided an affirmative answer to the above question and proposed the following property as ordinal analog of increasing differences: A function $F: S \times A \rightarrow R$ has the single-crossing property in $(s ; a)$ if $\forall a>a^{\prime}, s>s^{\prime}$,

$$
F\left(s^{\prime}, a\right)-F\left(s^{\prime}, a^{\prime}\right) \geq 0 \Rightarrow F(s, a)-F\left(s, a^{\prime}\right) \geq 0 .
$$

The single-crossing property is strict if $F\left(s^{\prime}, a\right)-F\left(s^{\prime}, a^{\prime}\right) \geq 0 \Rightarrow F(s, a)-F\left(s, a^{\prime}\right)>0$.

Unlike the property of increasing differences, the single-crossing property discriminates between

\footnotetext{
${ }^{23}$ These three assumptions say, respectively, that a firm's equilibrium profit from the product market decreases with own cost, increases with rival's cost, as well as with a common cost increase.

${ }^{24}$ Observe that the convenient cross partial test cannot be used here because the payoff has a kink along the diagonal $x_{1}=x_{2}$. So one must use Equation 2.

${ }^{25}$ The arguments used in this model constitute a general approach for constructing strategic games with a priori identical players but only asymmetric equilibria, that is, models with strategic endogenous heterogeneity.
} 
the two variables (action and parameter). In other words, satisfying the single-crossing property in $(s ; a)$ need not imply the same property in $(a ; s)$.

It is straightforward to verify, directly from the definitions, that

(i) the single-crossing property is more general than increasing differences, in that a function that satisfies the latter necessarily satisfies the former.

(ii) if $F$ satisfies the single-crossing property, then for any strictly increasing transformation $g, g \circ F$ will also satisfy the single-crossing property (so that this is an ordinal property).

The economic interpretation of this ordinal property is natural, but in terms of a limited form of complementarity. Thus, the single-crossing property says that, if a given increase in the decision variable is profitable when the parameter is low, the same increase will continue to be profitable when the parameter is high. Unlike increasing differences, though, it does not require the latter profitability level to exceed the former.

An important generalization ${ }^{26}$ of Topkis's result is due to Milgrom and Shannon (1994).

Theorem 6. Consider Problem (1) with $S, A \subset R$ and assume that (i) $\mathrm{F}$ has the single-crossing property in $(a ; s)$, and (ii) $A_{s}=[g(s), h(s)]$ where $h, g: S \rightarrow R$ are increasing functions with $g \leq h$. Then the maximal and minimal selections of $a^{*}(s), \bar{a}(s)$, and $a(s)$, are increasing functions. Furthermore, if (i) is strict, then every selection of $a^{*}(s)$ is increasing.

PROOF. Repeat the contradiction argument of Theorem 1 up to Equation 3, replacing it by

$$
F[s, \bar{a}(s)]-F\left[s, \bar{a}\left(s^{\prime}\right)\right] \geq 0 \Rightarrow F\left[s^{\prime}, \bar{a}(s)\right]-F\left[s^{\prime}, \bar{a}\left(s^{\prime}\right)\right] \geq 0,
$$

that is, the single-crossing property. This implies that $\bar{a}(s) \in a^{*}\left(s^{\prime}\right)$. Together with the contradiction hypothesis $\bar{a}\left(s^{\prime}\right)<\bar{a}(s)$, this is a contradiction to $\bar{a}(\cdot)$ being the maximal selection of $a^{*}(s)$. A similar change works for the case of strict single-crossing property. $Q E D$.

While the scope of this theory of monotone comparative statics is considerably enlarged by this result, as will be argued below via numerous examples, new difficulties appear with the verification of the underlying ordinal conditions in practical applications of the result. In particular, no differential characterization of these properties is available for smooth functions. Besides using the definition directly, which is often quite involved even for relatively simple problems, Milgrom and Shannon (1994) developed the following relatively practical test based on the well-known Spence-Mirrlees condition (defined by Equation 7 below).

THEOREM 7. Let $F: R^{3} \rightarrow R$ be continuously differentiable and $F_{2}(a, b, s) \neq 0 . F(a, h(a), s)$ satisfies the single-crossing property in $(a ; s)$ for all functions $h: R \rightarrow R$ if and only if

$$
F_{1}(a, b, s) /\left|F_{2}(a, b, s)\right| \quad \text { is increasing in } s .
$$

The proof is omitted (not insightful). This test is used in applications by verifying Equation 7 and concluding that the objective $F(a, h(a), s)$ satisfies the single-crossing property in $(a ; s)$ for a suitable choice of function $h$ (often the identity function, see examples below.)

\footnotetext{
${ }^{26}$ The version we state here is a special case of the original theorem, which captures its essential aspects, particularly as far as economic applications are concerned.
} 


\section{Some Economic Applications}

In order to facilitate comparison with the cardinal complementarity conditions, we derive assumptions on the primitives of some of the models from our previous examples that imply that the ordinal complementarity conditions hold. These examples confirm, indeed, that the scope of the theory has been significantly expanded by the ordinal complementarity notion. The scope of games with strategic complementarity has also been broadened because Theorems 4-7 continue to hold if supermodularity is replaced by the single-crossing property. ${ }^{27}$

\section{Cournot Duopoly}

The payoff to firm 1 (say) is $\Pi_{1}\left(q_{1}, q_{2}\right)=q_{1} P\left(q_{1}+q_{2}-C_{1}\left(q_{1}\right)\right.$. To show that firm 1's reaction correspondence is downward sloping, let $F(a, b, s)=b P(a+s)-C(b)$. Then $F_{1}(a, b, s)=b P^{\prime}(a+s)$, $F_{2}(a, b, s)=P(a+s)-C^{\prime}(a)$, and

$$
\frac{\partial F_{1}(a, b, s) /\left|F_{2}(a, b, s)\right|}{\partial s}=b \frac{\left[P(a+s)-C^{\prime}(b)\right] P^{\prime \prime}(a+s)-P^{\prime 2}(a+s)}{\left[P(a+s)-C^{\prime}(b)\right]^{2}} .
$$

Then Equation 7 holds here if $\left[P(b+s)-C^{\prime}(a)\right] P^{\prime \prime}(b+s)-P^{\prime 2}(b+s) \leq 0$, for which a sufficient condition is $P(\cdot) P^{\prime \prime}(\cdot)-P^{\prime 2}(\cdot) \leq 0$, or the log concavity of $P(\cdot)$. Taking $h$ as the identity function in Theorem 7, we conclude that $F(a, a, s)=a P(a+s)-C(a)$ has the single-crossing property in $(a ; s)$, or that $\Pi_{i}\left(q_{1}, q_{2}\right)$ has the single-crossing property in $\left(q_{1} ; q_{2}\right)$. So the Cournot oligopoly is a game of strategic substitutes under very general conditions. Indeed, any concave demand function is log concave. Not being too convex is all that log concavity requires. The limit case is $P(q)=e^{-q}$, which is convex and log linear.

Amir (1996b) shows the same result using the definition of the single-crossing property directly. The proof is more involved but the result is a bit more general because the regularity conditions of Theorem 7 are not needed (see Appendix for the proof).

A partial duality is also of interest: If the cost function is identically zero, then firm $i$ 's profit function satisfies the strict single-crossing property in $\left(q_{i}, q_{-i}\right)$ if $P$ is $\log$ convex, in which case, the Cournot oligopoly is a game of strategic complements! More precisely, profits are then easily seen to be $\log$ supermodular because $\log$ convexity of $P(\cdot)$ is equivalent to the $\log$ supermodularity of $P\left(q_{i}+\right.$ $\left.q_{-i}\right)$, and $\log F_{i}\left(q_{i}, q_{-i}\right)=\log q_{i}+\log P\left(q_{i}+q_{-i}\right)$, with the first term being trivially supermodular. Hence, the $n$-firm oligopoly is a quasi-supermodular game (with the original or natural output sets). Log convexity requires a strong form of convexity, the limit case being also $P(q)=e^{-q}$, and the typical example being the hyperbolic demand, for example, $P(q)=1 / q^{\alpha}$. For details, see Amir (1996b).

\section{Monopoly Pass-Through}

As seen in section 2, $\Pi$ has increasing differences if $D^{\prime} \leq 0$ while log $\Pi$ always has increasing differences. Because the exponential (exp) is a monotone transformation and $\exp (\log )$ is the identity function, it follows that $\Pi$ also satisfies the single-crossing property for all $D$ functions because the latter property is preserved by monotone transformations.

\footnotetext{
${ }^{27}$ One property of such games that does not extend to the ordinal realm is the fact that such games also have strategic complementarity in mixed strategies (Echenique 2001b).
} 


\section{Consumer Theory}

Consider the problem stated in the consumer theory example in Section 2. To ensure, using Theorem 7, that the objective in Equation 5 satisfies the single-crossing property in $\left(x_{1}, m\right)$ so that good 1 is a normal good, let $F(a, b, s)=U\left(b,\left(s-p_{1} a\right) / p_{2}\right)$. Then $F_{1}(a, b, s)=\left(-p_{1} / p_{2}\right) U_{2}\left(b,\left(s-p_{1} a\right) /\right.$ $\left.p_{2}\right), F_{2}(a, b, s)=U_{1}\left(b,\left(s-p_{1} a\right) / p_{2}\right)$, and Equation 7 holds here if

$$
U_{2} U_{21}-U_{1} U_{22} \geq 0, \quad \forall x_{1}, x_{2} \geq 0,
$$

or, equivalently, if the ratio of marginal utilities $U_{1}\left(x_{1}, x_{2}\right) / U_{2}\left(x_{1}, x_{2}\right)$ is increasing ${ }^{28}$ in $x_{2}$. From Theorem 7, with $h$ as the identity function, we conclude that good 1 is normal.

As a further comparative illustration, consider the specific example where $U\left(x_{1}, x_{2}\right)=x_{1}^{2} x_{2}^{2}$. Evaluating the various partials, we have $U_{1}=2 x_{1} x_{2}^{2}, U_{2}=2 x_{1}^{2} x_{2}, U_{21}=4 x_{1} x_{2}$, and $U_{22}=2 x_{1}^{2}$. It is easily seen by inspection that $U$ is strictly quasi-concave in $\left(x_{1}, x_{2}\right)$, though convex in each variable separately. Using the sufficient Condition 6, we can conclude that $x_{1}$ is a normal good if $p_{2} U_{21}-p_{1} U_{22}=4 x_{1} x_{2} p_{2}-$ $2 x_{1}^{2} p_{1} \geq 0$ or if $2 x_{2} p_{2}-x_{1} p_{1} \geq 0$, which clearly fails to hold globally (e.g., for sufficiently small values of $x_{1}$ ). Hence, we cannot conclude from Condition 6 that $x_{1}$ is a normal good at all price vectors.

However, using Condition 8 , we have $U_{2} U_{21}-U_{1} U_{22}=8 x_{1}^{3} x_{2}^{2}-4 x_{1}^{3} x_{2}^{2}=4 x_{1}^{3} x_{2}^{2} \geq 0$, so that we can directly conclude that $x_{1}$ is a normal good at all price vectors. To verify, an elementary computation yields the familiar Cobb-Douglas demand functions $x_{1}^{*}=m / 2 p_{1}$ and $x_{2}^{*}=m / 2 p_{2}$, so that both goods are indeed normal. ${ }^{29}$

\section{Bertrand Competition}

Consider the model of price competition stated in the Berthand competition example in Section 3, but with each firm's cost function being convex. Milgrom and Shannon (1994) show that firm $i$ 's profit function then satisfies the single-crossing property in $\left(p_{i}, p_{-i}\right)$. A sense of the difficulty in establishing the single-crossing property can be gained by looking at this proof.

\section{Nonrenewable Resource Extraction}

Consider the two-period problem of resource extraction with stock-dependent utility, no discount, and with the notation of Growth Theory:

$$
\max \sum_{t=1}^{2} \log \left[x_{t}\left(x_{t}-y_{t}\right)+1\right] \quad \text { subject to } x_{t+1}=y_{t} \quad \text { and } \quad 0 \leq y_{t} \leq x_{t} .
$$

Since the one-period value function is clearly $V_{1}(x)=\log \left(y^{2}+1\right)$, the two-period value function $V_{2}$ satisfies

$$
\left.V_{2}(x)=\max \left\{\log [x(x-y)+1]+\log \left(y^{2}+1\right)\right]: y \in[0, x]\right\} .
$$

\footnotetext{
${ }^{28}$ Because the first-order condition here is the familiar $p_{1} / p_{2}=U_{1} / U_{2}$, one can obtain the sufficient condition for ordinal complementarity from its cardinal analog by substituting the first-order relation into the latter. While this connection fails to hold as a general result, it does provide a good intuitive and informal way of relating the two sets of conditions.

${ }^{29}$ It is important to observe that, while Condition 6 is necessary and sufficient for cardinal complementarity, Condition 8 is sufficient, but not necessary, for ordinal complementarity. Either condition may hold while the other fails to hold for particular examples. In other words, we can also provide the reverse illustration to the one just provided. For Bertrand oligopoly, this is done in Amir and Grilo (2003).
} 
The optimal savings correspondence is (for details, see Amir 1996a, p. 138):

$$
y^{*}(x)= \begin{cases}\{0,1\}, & \text { if } \quad 0 \leq x \leq 1, \\ \frac{1}{3}\left[x+\frac{1}{x}+\frac{1}{x}\left(x^{4}-x^{2}+1\right)^{1 / 2}\right], & \text { if } \quad x \geq 1 .\end{cases}
$$

The maximal and minimal selections of $y^{*}(x)$ are both increasing in $x$. Yet, the objective in Equation 9 is submodular in $(x, y)$ if $x \leq 1$ ! Indeed, the cross partial of the objective is equal to $x(x-1) /[x(x-y)+$ $1]^{2}$, which is $\leq 0$ if and only if $x \leq 1$. So the objective does not satisfy the single-crossing property for $x \leq 1$. The feasible set $[0, x]$ satisfies our condition.

This example establishes, in particular, that the assumptions in Milgrom-Shannon's Theorem are not necessary for the monotonicity conclusion. This is an important point to clarify because the Milgrom-Shannon Theorem is actually stated with necessary and sufficient conditions for monotonicity of the argmax with respect to both an ordinary parameter (such as $s$ here) and the constraint set itself. Thus, this example, shows nicely that the conditions of the Theorem are not necessary if monotonicity is to hold only with respect to the parameter, as in the present treatment, given a constraint set satisfying Assumption (ii) of Theorem 1.

There are other studies, mostly in industrial organization, in addition to those already mentioned, which make use of the theory of supermodularity. These include Bagwell and Ramey (1994), Friedman and Johnson (1997), Peitz (2000), and Athey and Schmutzler (2001). The analysis of endogenous timing in two-player games according to a natural scheme proposed by Hamilton and Slutsky (1990) is also best approached with the tools of supermodularity (see, e.g., Amir and Grilo 1999). More applications are given in Milgrom and Shannon (1994), Shannon (1995), Topkis (1998), and Vives (1999).

\section{The Multidimensional Case}

Our simplified treatment is extended here to the multidimensional case. This presentation still falls short of the full generality of the theory. We begin with the requisite preliminaries. Let $R^{n}$ denote $n$-dimensional Euclidean space. Given $x=\left(x_{1}, x_{2}, \ldots, x_{n}\right)$ and $y=\left(y_{1}, y_{2}, \ldots, y_{n}\right)$ in $R^{n}$, denote by $x \vee y$ and $x \wedge y$ the coordinatewise supremum and infinum of $x$ and $y$,

$$
x \vee y=\left(\max \left\{x_{1}, y_{1}\right\}, \ldots, \max \left\{x_{n}, y_{n}\right\}\right), \quad \text { and } x \wedge y=\left(\min \left\{x_{1}, y_{1}\right\}, \ldots, \min \left\{x_{n}, y_{n}\right\}\right) .
$$

The inequality $x \geq y$ means $x_{i} \geq y_{i}$ for all $i$ (coordinatewise order), while $x>y$ means $x \geq y$ and $x_{i}>$ $y_{i}$ for at least one $i$. A rectangle in $R^{n}$ is a Cartesian product of $n$ real intervals. Thus, a compact rectangle in $R^{n}$ is any set of the form $X_{i=1}^{i=n}\left[a_{i}, b_{i}\right]$ for some reals $a_{i}, b_{i}$. Let $S$ (parameter set) and $A$ (action set) be rectangles in $R^{l}$ and $R^{m}$, respectively.

\section{Cardinal Complementarity Conditions}

This part is based on Topkis (1978). A function $F: A \rightarrow R$ is (strictly) supermodular if

$$
F\left(a \wedge a^{\prime}\right)+F\left(a \vee a^{\prime}\right)(>) \geq F(a)+F\left(a^{\prime}\right), \quad \forall a, a^{\prime} \in A .
$$

If $F$ is smooth, $F$ is supermodular if and only if $\partial^{2} F(a) / \partial a_{i} \partial a_{j} \geq 0, \forall i \neq j$, that is, if and only if all the nondiagonal terms of the Hessian matrix of $F$ are positive (no restrictions on the diagonal terms). 
A function $F: S \times A \rightarrow R$ has (strictly) increasing differences in $(s, a)$ if

$$
F(s, a)-F\left(s, a^{\prime}\right)(>) \geq F\left(s^{\prime}, a\right)-F\left(s^{\prime}, a^{\prime}\right), \quad \forall a>a^{\prime}, s>s^{\prime},
$$

or in other words, if the difference $F(\cdot, a)-F\left(\cdot, a^{\prime}\right)$ is an increasing function. If $F$ is smooth, Equation 11 is equivalent to $\partial^{2} F(a) / \partial a_{i} \partial s_{j} \geq 0$, for all $i, j$. Note that there are no restrictions here on partials of the form $\partial^{2} F(s, a) / \partial a_{i} \partial a_{j}$ or $\partial^{2} F(s, a) / \partial s_{i} \partial s_{j}$.

For the scalar case $(l=m=1)$ treated earlier, supermodularity and increasing differences are easily seen to be equivalent for $f$, as both are then characterized by $\partial^{2} F(s, a) / \partial a \partial s \geq 0$. Supermodularity of a function $f$ of $n$ variables is equivalent to increasing differences or supermodularity of $F$ with respect to all pairs of variables. This is a simple test, as it reduces to checking all pairwise relations only. Thus, the complementarity interpretation of supermodularity and increasing differences extends to the multidimensional case.

A simplified multidimensional version of Topkis's Monotonicity Theorem is now given. This is still a special case of the original result, particularly with regard to the structure of $A_{s}$. The proof is a simple extension of the proof of Theorem 1.

THEOREM 8. Assume

(i) $F$ is supermodular in $a$ for each fixed $s$,

(ii) $F$ has increasing differences in $(s, a)$, and

(iii) $A_{s}=\mathrm{X}_{i=1}^{i=m}\left[g_{i}(s), h_{i}(s)\right]$, where $h_{i}, g_{i}: S \rightarrow R$ are increasing functions with $g_{i} \leq h_{i}$. Then the maximal and minimal selections ${ }^{30}$ of $\mathrm{a}^{*}(\mathrm{~s})$ are increasing functions. Furthermore, if (ii) is strict, then every selection of $a^{*}(s)$ is increasing.

To relate this result to Theorem 1, observe that the added assumption here, supermodularity of $F$ in $a$, is always trivially satisfied if $a$ is scalar; hence, its omission in Theorem 1 . If $a$ is a vector, it is clear, intuitively, that such an assumption is needed to insure that the direct increases in the coordinates of $a$ triggered by an increase in $s$ are mutually reinforcing. ${ }^{31}$

The following is a smooth version of Topkis's Theorem, based on Lemma 1 and the remarks following Equation 11.

THEOREM 9. Theorem 8 continues to hold if (i) and (ii) are replaced, respectively, by $\partial^{2} F(a) /$ $\partial a_{i} \partial a_{j} \geq 0, \forall i \neq j$ and $\partial^{2} F(a) / \partial a_{i} \partial s_{j} \geq 0, \forall i, j$, with (iii) kept as is.

This smooth version makes transparent the fact that there are no restrictions on partials of the form $\partial^{2} F(a) / \partial s_{i} \partial s_{j}$. A sufficient condition for (i) and (ii) is to require $F$ to be supermodular in the vector $(a, s)$, which would unnecessarily require the latter partials to be $\geq 0$.

Theorem 8 admits a natural dual for decreasing argmaxes.

THEOREM 10. If "increasing" is replaced by "decreasing" in Theorem 8's (ii) and (iii), with all else kept as is, the two given conclusions hold with "increasing" replaced by "decreasing."

Of the other results from Topkis (1978), the following has proved useful in a number of applications (see, e.g., Amir, Mirman, and Perkins 1991; Amir 1996a).

THEOREM 11. Let $a=\left(a^{j}, a^{-j}\right) \in A$ and assume $F: A \rightarrow R$ is supermodular in $a$, then $V\left(a^{-j}\right) \triangleq$ $\max _{a^{\mathrm{j}}} F(a)$ is supermodular in $a^{-j}$.

\footnotetext{
30 These are always well-defined, single-valued functions under Condition (i) of the Theorem.

${ }^{31}$ Without this assumption, one cannot guarantee a monotone comparative statics conclusion for all the coordinates of the vector $a$. See Samuelson (1974) for his coffee-tea paradox.
} 
In other words, this result says that maximization of a supermodular objective with respect to a subset of the variables preserves supermodularity with respect to the remaining variables. The use of the above results is illustrated in the following model.

\section{Monopoly with $R \& D$ and Advertising}

Consider a monopolist with three choice variables: price $p$, advertizing $a$, and investment in process $\mathrm{R} \& \mathrm{D} x$. The market demand function is given by $D(p, a, \theta)$, where $\theta \in[0,1]$ is a parameter capturing business cycle effects, with higher $\theta$ meaning better macroeconomic conditions. Assume $D$ satisfies $^{32}$

$$
D_{p}<0, \quad D_{a}>0, \quad D_{\theta}>0, \quad D_{a}+p D_{a p}<0, \quad D_{\theta}+p D_{p \theta}<0, \quad D_{a \theta}>0 .
$$

The first three parts of (12) express, respectively, the natural assumptions that sales decrease with price and increase with advertizing and with (upwings in) the business cycle. The fourth part says that revenue has increasing differences in $(p, a)$, which requires a strongly submodular demand in $(p, a)$, here as $D_{a}>0$, and is a rather restrictive condition. A similar comment applies to the fifth part of (12). The sixth part says that the extra sales generated by higher advertizing increase with the business cycle, a reasonable property.

The firm has a constant unit cost, $C(x, \beta)$, depending on its process R\&D investment $x$ and on an interindustry spillover level $\beta \in[0,1]$. We assume that

$$
C_{x}<0, \quad C_{\beta}<0 \quad \text { and } \quad C_{\beta x} \leq 0 .
$$

The last condition may be interpreted as saying that the marginal cost reduction is increasing in the level of spillovers. The overall profit function of the firm is

$$
\tilde{\Pi}(p, a, x ; \theta, \beta)=[p-C(x, \beta)] D(p, a, \theta)-x .
$$

We first show that the optimal decision vector $\left(-p^{*}, a^{*}, x^{*}\right)$ is increasing in $(\theta, \beta)$. To this end, observe that, in view of Assumptions 12 and 13, $\tilde{\Pi}$ is supermodular in $(-p, a, x)$ as

$$
\tilde{\Pi}_{(-p) a}=-\tilde{\Pi}_{p a}=-D_{a}-p D_{a p}>0, \quad \tilde{\Pi}_{(-p) x}=-\tilde{\Pi}_{p x}=C_{x} D_{p}>0, \quad \text { and } \quad \tilde{\Pi}_{a x}=-C_{x} D_{a}>0,
$$

and $\tilde{\Pi}$ has increasing differences in $[(-p, a, x),(\theta, \beta)]$ because

$$
\begin{aligned}
\tilde{\Pi}_{(-p) \theta} & =-\tilde{\Pi}_{p \theta}=-D_{\theta}-p D_{p \theta}>0, \quad \tilde{\Pi}_{(-p) \beta}=-\tilde{\Pi}_{p \beta}=C_{\beta} D_{p} \geq 0 ; \quad \tilde{\Pi}_{a \beta}=-C_{\beta} D_{a}>0, \\
\tilde{\Pi}_{a \theta} & =(p-C) D_{a \theta}>0 ; \quad \text { and } \quad \tilde{\Pi}_{x \theta}=-C_{x} D_{\theta}>0, \quad \tilde{\Pi}_{x \beta}=-C_{x \beta} D>0 .
\end{aligned}
$$

This establishes that, as $\theta$ and $\beta$ increase, the optimal advertizing and $R \& D$ investment levels increase while the optimal price decreases (in the sense that all the selections from the three decisions are monotonic as indicated).

Next, we address a somewhat different issue: relating short- and long-run responses to a given change. Suppose that, in the short run, technology or R\&D investment is fixed. We can still conclude from our previous analysis that the optimal price and advertizing will then react as before to changes in $\theta$ and $\beta$. A question of interest is: Are these reactions greater in the short run or in the long run? The answer is that they are greater in the long run because, in the long run, a given change in $\theta$ and

\footnotetext{
32 Observe that the analysis below will not rely on any concavity or interiority assumptions whatsoever. Furthermore, while the smoothness assumptions are convenient to express complementarity conditions in intuitive ways, they are not needed in any crucial sense for the underlying analysis and results.
} 
$\beta$ has all the short-run effects plus the direct effect of increasing $R \& D$ investment $x$, which, in turn, has the known indirect effects of increasing $(-p, a)$. This is a simple expression of LeChatelier's principle (Milgrom and Roberts 1995b).

Last, we show the optimal profit function $\Pi *(\theta, \beta) \triangleq \arg \max \{\Pi(p, a, x ; \theta, \beta): p, a, x \geq 0\}$ is supermodular in $(\theta, \beta)$. To this end, we invoke Theorem 11 as follows. $\Pi(p, a, x ; \theta, \beta)$ is supermodular in $(p, a, x ; \theta, \beta)$ in view of Assumptions 12 and 13 and the fact that $\Pi_{\theta \beta}=-C_{\beta} D_{\theta}>0$. (In other words, given the latter computation, the cross partial of $\Pi$ with respect to any two of the five variables is $\geq 0$ ). Hence, because maximization preserves supermodularity, $\Pi^{*}(\theta, \beta)$ is supermodular in $(\theta, \beta)$. The interpretation is that the marginal profitability of higher $R \& D$ investment increases with (upswings in) the business cycle, again, a reasonable property.

\section{Ordinal Complementarity Conditions}

The ordinal versions of the multidimensional complementarity conditions, due to Milgrom and Shannon (1994), are as follows (with the same spaces and notation as above).

A function $f: A \rightarrow R$ is quasi-supermodular if $\forall a, a^{\prime} \in A$,

$$
F(a) \geq F\left(a \wedge a^{\prime}\right) \Rightarrow F\left(a \vee a^{\prime}\right) \geq F\left(a^{\prime}\right) \text { and } F(a)>F\left(a \wedge a^{\prime}\right) \Rightarrow F\left(a \vee a^{\prime}\right)>F\left(a^{\prime}\right) \text {. }
$$

A function $f: S \times A \rightarrow R$ has the single-crossing property in $(a ; s)$ if $\forall a^{\prime}>a, s^{\prime}>s$,

$$
\begin{aligned}
F\left(s, a^{\prime}\right)-F(s, a) \geq 0 & \Rightarrow F\left(s^{\prime}, a^{\prime}\right)-F\left(s^{\prime}, a\right) \geq 0, \quad \text { and } \\
F\left(s, a^{\prime}\right)-F(s, a)>0 & \Rightarrow F\left(s^{\prime}, a^{\prime}\right)-F\left(s^{\prime}, a\right)>0 .
\end{aligned}
$$

The single-crossing property is strict if $F\left(s, a^{\prime}\right)-F(s, a) \geq 0 \Rightarrow F\left(s^{\prime}, a^{\prime}\right)-F\left(s^{\prime}, a\right)>0$.

Recall that the single-crossing property is not symmetric with respect to the two variables, in that it may hold for $(a ; s)$ and not for $(\mathrm{s} ; a)$. On the other hand, quasi-supermodularity in $a$ treats all coordinates of $a$ symmetrically, and implies the single-crossing property in both $\left(a_{i} ; a_{j}\right)$ and in $\left(a_{j} ; a_{i}\right)$ for every $i \neq j$.

A simplified version of the main result of Milgrom and Shannon (1994) is:

THEOREM 12. The conclusions of Theorem 8 hold if supermodularity is replaced by quasisupermodularity and (strict) increasing differences by the (strict) single-crossing property.

\section{A Comparative Discussion}

Milgrom and Shannon (1994) also show that all the above results shown in section 3 for supermodular games also hold for quasi-supermodular games (i.e., games satisfying the ordinal complementarity conditions). Indeed, these results actually rely on the monotonicity of the bestresponse correspondences only, for which the ordinal conditions suffice.

Besides the advantage of being much easier to verify in actual applications, the cardinal complementarity conditions sometimes lead to some desirable properties that are not satisfied under the ordinal conditions. In particular, the following two differences between the two notions are often of critical importance in many applications: (i) The single-crossing condition is not preserved by addition and (ii) Theorem 11 need not hold if supermodularity is replaced by quasi-supermodularity. Thus, maximization of a quasi-supermodular objective with respect to a subset of the variables need not give rise to a quasi-supermodular value function (with respect to the remaining variables).

Although the ordinal conditions are more general than the cardinal conditions, in a typical 
application with the objective function being a sum or other composite of primitive functions, the respective minimally sufficient conditions on these primitives that imply the two types of conditions are generally not comparable. For a detailed investigation of this point centering on Cournot oligopoly, see Amir (2004).

There are some interesting analogies between supermodularity and quasi-supermodularity, on the other hand, and concavity and quasi-concavity on the other hand. Indeed, in both cases,

(i) The ordinal condition is more general than the cardinal condition.

(ii) The cardinal property survives addition while the ordinal property need not. This is a key difference in many economic applications because objective functions often take the form of a sum of some benefit terms and some cost terms. ${ }^{33}$ In such cases, the cardinal notions often turn out to be critically needed.

(iii) The cardinal property is conveniently verified via a set of inequalities on the Hessian matrix while the ordinal property requires a more difficult test.

(iv) The cardinal property is preserved by partial maximization (Theorem 11), while the ordinal property need not be.

\section{Other Aspects of the Theory}

Several other relevant aspects of the theory under consideration have not been covered in the present survey. In this section, we provide a nonexhaustive list of these aspects and a brief summary. Further details may be found in the given references.

\section{Parametric Optimization Under Uncertainty}

Athey (2001, 2002) develops several different general results dealing with monotone comparative statics and preservation of complementarity conditions for objective functions that are represented as the integral of a multivariate function against a transition probability (or a family of probability distributions). This work provides a unifying framework that encompasses most results on comparative statics under uncertainty in economics, as well as a new existence result for Bayesian games (such as auctions) in monotone strategies.

\section{Critical Sufficient Conditions}

As both Topkis's Theorem and Milgrom-Shannon's Theorem provide sufficient conditions for increasing optimal argmaxes, it is desirable to investigate how close to necessary these conditions are. The idea is to consider families of problems obtained by varying some model primitives such as a set of parameters. A condition is critical (with respect to a family of models) if, whenever it fails, the monotonicity conclusion will fail for some specific model from the designated family (see Milgrom and Shannon 1994; Milgrom and Roberts 1994a). For the model of Bertrand competition with constant unit costs, allowing the latter parameter to vary - thereby defining a firm - is a natural way of specifying the relevant family. See Topkis (1995) and Amir and Grilo (2003).

\footnotetext{
${ }^{33}$ In dynamic optimization problems, the objective to which Topkis's Theorem is applied is the right-hand side of the Bellman
} equation, a sum of today's reward and the value of the remainder (see the growth theory example in Section 2). 


\section{Strict Monotonicity of Argmaxes}

In some applications, it may be desirable to extend the conclusion of (weakly) increasing argmaxes to one of strict monotonicity. This requires two new assumptions: (i) smoothness of the objective function, (ii) a strengthening of the assumption of increasing differences to this property: The partial of the objective function with respect to the decision must be strictly (not weakly, as in Topkis's work) increasing in the parameter. See Amir (1996a), Edlin and Shannon (1998), or Topkis (1998, p. 71).

\section{Macroeconomics}

Models of Keynesian-type coordination failure can be usefully analyzed as supermodular games with multiple equilibria. See Cooper and John (1988) and Cooper (1999). A recent strand of literature deals with dynamic equilibrium models using super modularity techniques (Datta, Mirman, and Reffett 2002).

\section{Institutional Complementarity}

A rather different application of this framework is to study general socioeconomic systems using the underlying notions of complementarity as interpretative and descriptive properties in a much more precise manner than is typically done in related work. This general area of application is somewhat different from the material covered in the present survey in that, most often, no specific models of the issues under consideration are developed. Rather, the framework of economic complementarities is invoked to explain the observed presence of certain clusters in firms' strategies, institutional arrangements and other aspects of socioeconomic organization that are mutually reinforcing. See Milgrom and Roberts (1990a, 1995a) and Hall and Soskice (2001).

\section{Appendix: Proofs}

We provide the proofs of the various results stated in the main text, in order of appearance.

Lemma 2. In Cournot oligopoly, assume that $P(\cdot)$ is strictly decreasing and log concave and each firm's cost function is strictly increasing and lower semicontinuous. Then the profit function satisfies the single-crossing property in $\left(q_{i}, q_{-i}\right)$.

Proof. We need to show that, for any $q_{i}^{\prime}>q_{i}$ and $q_{-i}^{\prime}>q_{-i}$,

$$
q_{i}^{\prime} P\left(q_{i}^{\prime}+q_{-i}\right)-C_{i}\left(q_{i}^{\prime}\right) \leq q_{i} P\left(q_{i}+q_{-i}\right)-C_{i}\left(q_{i}\right) \Rightarrow q_{i}^{\prime} P\left(q_{i}^{\prime}+q_{-i}^{\prime}\right)-C_{i}\left(q_{i}^{\prime}\right)<q_{i} P\left(q_{i}+q_{-i}^{\prime}\right)-C_{i}\left(q_{i}\right) .
$$

Logconcavity of $P(\cdot)$ is equivalent to $\log$ submodularity of $P\left(q_{i}+q_{-i}\right)$ in $\left(q_{i}, q_{-i}\right)$, that is,

$$
\log P\left(q_{i}^{\prime}+q_{-i}^{\prime}\right)+\log P\left(q_{i}+q_{-i}\right) \leq \log P\left(q_{i}^{\prime}+q_{-i}\right)+\log P\left(q_{i}+q_{-i}^{\prime}\right),
$$

or

$$
P\left(q_{i}^{\prime}+q_{-i}^{\prime}\right) P\left(q_{i}+q_{-i}\right) \leq P\left(q_{i}^{\prime}+q_{-i}\right) P\left(q_{i}+q_{-i}^{\prime}\right) .
$$

With the left-hand side of Equation A1 as a starting point,

$$
q_{i}^{\prime} P\left(q_{i}^{\prime}+q_{-i}\right)-C_{i}\left(q_{i}^{\prime}\right) \leq q_{i} P\left(q_{i}+q_{-i}\right)-C_{i}\left(q_{i}\right) \leq q_{i} P\left(\frac{\left.q_{i}^{\prime}+q_{-i}\right) P\left(q_{i}+q_{-i}^{\prime}\right)}{P\left(q_{i}^{\prime}+q_{-i}^{\prime}\right)}-C_{i}\left(q_{i}\right),\right.
$$

by Equation A2. Multiplying across by $P\left(q_{i}^{\prime}+q_{-i}^{\prime}\right) / P\left(q_{i}^{\prime}+q_{-i}\right)$,

$$
q_{i}^{\prime} P\left(q_{i}^{\prime}+q_{-i}^{\prime}\right)-\frac{P\left(q_{i}^{\prime}+q_{-i}^{\prime}\right)}{P\left(q_{i}^{\prime}+q_{-i}\right)} C_{i}\left(q_{i}^{\prime}\right) \leq q_{i} P\left(q_{i}+q_{-i}^{\prime}\right)-\frac{P\left(q_{i}^{\prime}+q_{-i}^{\prime}\right)}{P\left(q_{i}^{\prime}+q_{-i}\right)} C_{i}\left(q_{i}\right) .
$$

Because $C_{i}\left(q_{i}^{\prime}\right)>C_{i}\left(q_{i}\right)$ and $P\left(q_{i}^{\prime}+q_{-i}^{\prime}\right)<P\left(q_{i}^{\prime}+q_{-i}\right)$, it follows that 


$$
q_{i}^{\prime} P\left(q_{i}^{\prime}+q_{-i}^{\prime}\right)-C_{i}\left(q_{i}^{\prime}\right)<q_{i} P\left(q_{i}+q_{-i}^{\prime}\right)-C_{i}\left(q_{i}\right) .
$$

Because Equation A1 holds, the proof is complete. QED.

\section{References}

Amir, Rabah. 1996a. Sensitivity analysis in multisector optimal economic dynamics. Journal of Mathematical Economics 25:123-41.

Amir, Rabah. 1996b. Cournot oligopoly and the theory of supermodular games. Games and Economic Behavior 15:132-48.

Amir, Rabah. 2004. Oridnal vs cardinal complementarity conditions: The case of Cournot oligopoly. Games and Economic Behavior. In press.

Amir, Rabah, and Isabel Grilo. 1999. Stackelberg versus Cournot equilibrium. Games and Economic Behavior 26:1-21.

Amir, Rabah, and Isabel Grilo. 2003. On complementarity conditions in Bertrand oligopoly. Economic Theory 22:227-32.

Amir, Rabah, and Val E. Lambson. 2000. On the effects of entry in Cournot markets. Review of Economic Studies 67:235-54.

Amir, Rabah, Leonard J. Mirman, and William R. Perkins. 1991. One-sector nonclassical optimal growth: Optimality conditions and comparative dynamics. International Economic Review 32:625-44.

Amir, Rabah, and John C. Wooders. 1999. Effects of one-way spillovers on firm size, industry price, welfare and R\&D cooperation. Journal of Economics and Management Strategy 8:223-49.

Amir, Rabah, and John C. Wooders. 2000. One-way spillovers, endogenous innovator/imitator roles and research joint ventures. Games and Economic Behavior 31:1-25.

Athey, Susan C. 2001. Single crossing properties and the existence of pure strategy equilibria in games of incomplete information. Econometrica 69:861-89.

Athey, Susan C. 2002. Monotone comparative statics under uncertainty. Quarterly Journal of Economics 117:187-223.

Athey, Susan C., and Armin Schmutzler. 2001. Investment and market dominance. RAND Journal of Economics 32:1-26.

Bagwell, Kyle, and Gary Ramey. 1994. Coordination economies, advertizing and search behavior in retail markets. American Economic Review 84:498-517.

Becker, Gary. 1973. A theory of marriage: Part I. Journal of Political Economy 81:813-46.

Bülow, Jeremy, John Geanakoplos, and Paul Klemperer. 1985. Multimarket oligopoly: Strategic substitutes and somplements. Journal of Political Economy 93:488-511.

Cooper, Russell. 1999. Coordination games: Complementarities and macroeconomics. Cambridge, UK: Cambridge University Press.

Cooper, Russell, and Andrew John. 1988. Coordinating coordination failures in Keynesian models. Quarterly Journal of Economics 103:441-63.

Datta, Manjira, Leonard Mirman, and Kevin Reffett. 2002. Existence and uniqueness in distorted dynamic economies with capital and labor. Journal of Economic Theory 103:377-410.

Diamond, Peter. 1982. Aggregate demand management in search equilibrium. Journal of Political Economy 90:881-94.

Echenique, Federico. 2001a. A characterization of strategic complementarities. Games and Economic Behavior 46:325-47.

Echenique, Federico. 2001b. Mixed equilibria in games of strategic complementarities. Working Paper, University of California at Berkeley.

Echenique, Federico. 2002. Comparative statics by adaptive dynamics and the correspondence principle. Econometrica 70:833-44.

Echenique, Federico, and Aaron Edlin. 2001. Mixed equilibria in games of strategic complements are unstable. Mimeo.

Edlin, Aaron, and Chris Shannon. 1998. Strict monotonicity in comparative statics. Journal of Economic Theory 89:201-19.

Friedman, Eric, and Simon Johnson. 1997. Dynamic monotonicity and comparative statics for real options. Journal of Economic Theory 75:104-21.

Fundenberg, Drew, and Jean Tirole. 1991. Game theory. Cambridge, MA: MIT Press.

Hall, Peter, and David Soskice, eds. 2001. Varieties of capitalism: The institutional foundations of comparative advantage. Volumes 1 and 2. Oxford, UK: Oxford University Press.

Hamilton, Jonathan, and Steven Slutsky. 1990. Endogenous timing in duopoly games: Stackelberg or Cournot equilibria. Games and Economic Behaviour 2:29-46.

Hoernig, Steffen. 2003. Existence of equilibrium and comparative statics in differentiated goods Cournot oligopolies. International Journal of Industrial Organization 21:989-1020.

Milgrom, Paul, and John Roberts. 1990a. The economics of modern manufacturing: Technology, strategy and organization. American Economic Review 80:511-28.

Milgrom, Paul, and John Roberts. 1990b. Rationalizability, learning, and equilibrium in games with strategic complementarities. Econometrica 58:1255-78.

Milgrom, Paul, and John Roberts. 1994a. Comparing equilibria. American Economic Review 84:441-59.

Milgrom, Paul, and John Roberts. 1994b. Understanding the Japanese economic system. Estudios Economicos 9:3-42. 
Milgrom, Paul, and John Roberts. 1995a. Complementarities and fit: Strategy, structure, and organizational change in manufacturing. Journal of Accounting and Economics 19:179-208.

Milgrom, Paul, and John Roberts. 1995b. The LeChatelier principle. American Economic Review 86:173-9.

Milgrom, Paul, and Christina Shannon. 1994. Monotone comparative statics. Econometrica 62:157-80.

Novshek, William. 1985. On the existence of Cournot equilibrium. Review of Economic Studies 52:85-98.

Peitz, Martin. 2000. Aggregation in a model of price competition. Journal of Economic Theory 90:1-38.

Samuelson, Paul. 1947. Foundations of economic analysis. Harvard Economic Studies, volume 80. Cambridge, MA: Harvard University Press.

Samuelson, Paul. 1974. Complementarity. Journal of Economic Literature 12:1255-89.

Seade, Jesus. 1980. On the effects of entry. Econometrica 48:479-89.

Shannon, Christina. 1995. Weak and strong monotone comparative statics. Economic Theory 5:209-27.

Tarski, Alfred. 1955. A lattice-theoretic fixed point theorem and its applications. Pacific Journal of Mathematics 5:285-309.

Topkis, M. Donald. 1968. Ordered optimal solutions. PhD dissertation, Stanford University.

Topkis, M. Donald. 1978. Minimizing a submodular function on a lattice. Operations Research 26:305-21.

Topkis, M. Donald. 1979. Equilibrium points in nonzero-sum n-person submodular games. SIAM Journal of Control and Optimization 17:773-87.

Topkis, M. Donald. 1995. Comparative statics of the firm. Journal of Economic Theory 67:370-401.

Topkis, M. Donald. 1998. Submodularity and complementarity. Princeton, NJ: Princeton University Press.

Vives, Xavier. 1990. Nash equilibrium with strategic complementarities. Journal of Mathematical Economics 19:305-21.

Vives, Xavier. 1999. Oligopoly pricing: Old ideas and new tools. Cambridge, MA: MIT Press. 
Reproduced with permission of the copyright owner. Further reproduction prohibited without permission. 